\title{
Review of the literature on anatomical variations of the trapezius muscle
}

\begin{abstract}
Introduction: The morphological diversity of the trapezius muscle is important, even if statistically these variations are rare. The authors of this work study anatomical variations of trapezius muscle in literature.
\end{abstract}

Material and methods: A systematic review of the literature using several databases has identified the main anatomical variations present on the trapezius muscle.

\begin{abstract}
Anatomical variations: The most frequently reported anatomical variations are agenesis and unilateral or bilateralaplasia, trapezius hypoplasia, the presence of accessory portions of the muscle, and accessory nerve abnormalities. However, variations in their bony insertions, and asymmetries predominate by their frequency in the trapezius muscle
\end{abstract}

Conclusion: The anatomical variations of trapezius muscle are real despite their rarity. They can have important functional consequences.

Keywords: trapezius, anatomical variations, review of the literature, functional consequences
Volume 4 Issue 5 - 2017

\author{
Philippe Manyacka MA Nyemb, ${ }^{1,2}$ Christian \\ Fontaine, ${ }^{3}$ Jean-Marc Ndoye ${ }^{4}$ \\ 'Laboratory of Anatomy and Organogenesis, Gaston Berger \\ University, Sénégal \\ ${ }^{2}$ General Surgery Service, Regional Hospital, Sénégal \\ ${ }^{3}$ Laboratory of Anatomy, University of Lille 2, France \\ ${ }^{4}$ Laboratory of Anatomy and Organogenesis, Cheikh Anta Diop \\ University, Sénégal
}

Correspondence: Philippe Manyacka MA Nyemb, Laboratory of Anatomy and Organogenesis, UFR 2S, Gaston Berger University, Road of Ngallèlle, 234 Saint-Louis, Sénégal, Tel (+22I) 7744543 13,Email phil_manyacka@yahoo.fr

Received: October 26, 2017 | Published: December 28 2017

\section{Introduction}

Muscle abnormalities of the posterior cervical triangle are rare and occur in only $2 \%$ of cases, including variations on the origin of the trapezius muscle. ${ }^{1,2}$ However, these abnormalities can lead to a restriction of the mobility of the upper limbs, head and neck. ${ }^{3,4}$ In addition, for the surgeon, these anatomical variations may be a contraindication to any flap trapezius surgery.

Through this work the authors propose to discuss the main anatomical variations reported by the literature concerning trapezius muscle.

\section{Surgical anatomy}

The trapezius muscle originates on the occipital bone, nuchal ligament, spinous process of the $7^{\text {th }}$ cervical vertebra (C7) and all of the thoracic vertebrae (Figure 1). It has three parts. The superior portion (descending fibers) terminates on the posterior margin and superior side of the lateral third of the clavicle. The transverse portion (middle fibers) terminates on the medial margin of the acromion and superior lip of the posterior border of the scapular spine. The inferior portion (ascending fibers) ends as an aponeurosis on the posterior edge of the scapular spine and a tubercle. The trapezius muscle is innervated by the accessory nerve and the cervical plexus. It helps to move the head (extension and rotation) and the shoulders (shrugging, lowering, posterior tipping, bringing the scapulae closer together).

According to the Mathes \& Nahai, ${ }^{5}$ the trapezius muscle receives type II vascularization with a primary pedicle from the transverse cervical artery and secondary pedicles issued from the occipital, intercostal and circumflex scapular perforating arteries.

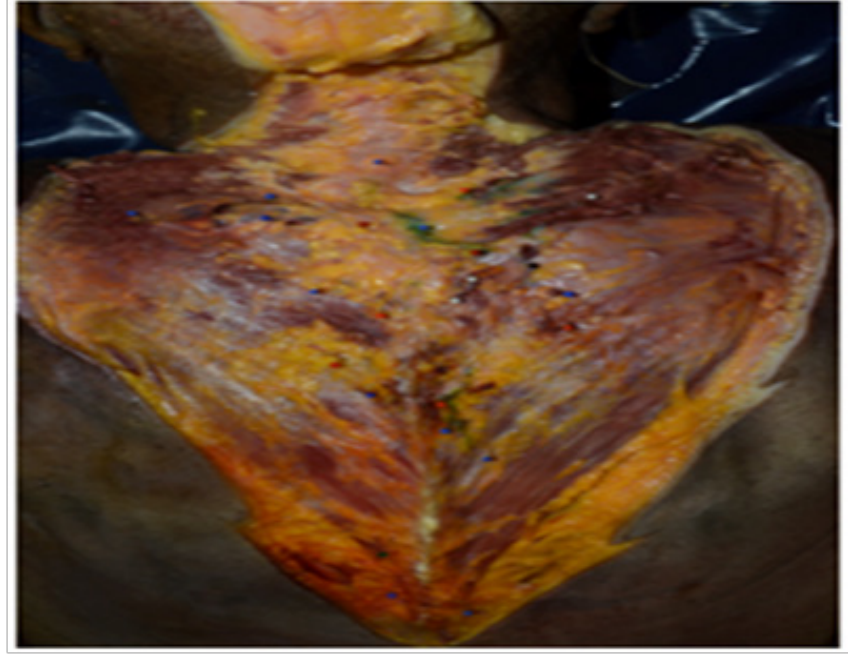

Figure I Dissection of a trapezius muscle.

The transverse cervical artery has a somewhat variable territory. However, its branches anastomose to those of the dorsal scapular artery to contribute to the vascularization of the trapezius, both in the muscle body itself and its integuments. Based on an anatomical study of 124 cadavers, ${ }^{6}$ the descending fibers receive branches from the occipital artery; the middle fibers and the lateral areas receive branches from the superficial branch of the transverse cervical artery; the ascending fibers receive branches from the dorsal scapular artery and the posterior intercostal arteries. Nevertheless, these vessels vastly anastomose with each other.

The occipital artery courses under the cranial portion of the 
trapezius muscle; some of its branches perforate the muscle layer to terminate in the integuments. The occipital artery has two terminal branches: a large medial branch that splits in the body of the trapezius muscle and anastomoses with the deep cervical artery and dorsal scapular artery, and a smaller, inconsistent lateral branch. ${ }^{7,8}$

At the nape of the neck, there are typically only two arterial trunks: a branch of the occipital artery and a branch of the deep cervical artery. The dermis and hypodermis networks are very dense in this area. The nape's skin has a reduced number of large arterial trunks but numerous arterioles. ${ }^{7}$

The dorsal region is mainly irrigated by the posterior intercostal arteries and dorsal scapular artery. Before entering the intercostal space, the posterior intercostal arteries give off a dorsal branch. The latter immediately courses posteriorly; once over the intervertebral foramen, it bifurcates into a spinal branch and a musculocutaneous branch. The musculocutaneous branch heads into the intertransverse space and then splits into a lateral muscular branch and a medial musculocutaneous branch. In the skin layer covering the vertebral grooves and the caudal portion of the trapezius muscle, there are two types of cutaneous arteries: posteromedial perforating arteries that stem from the medial branch of the musculocutaneous branch of the dorsal branch and posterolateral perforating arteries that stem from the lateral branch of this same branch.,

The superior cervical artery courses laterally to the levator scapulae muscle and the rhomboid muscles, before going medially to give off a (short) ascending branch and a (long) descending branch that pass next to the scapular spine. Its descending trajectory is superficial to the levator scapulae and rhomboid muscles, and next to a few branches of the external branch of the accessory nerve. At this level, its diameter is generally greater than $0.7 \mathrm{~mm} .{ }^{9}$

The dorsal scapular artery courses deep to the levator scapulae and rhomboid muscles. Branches then pierce the fascia separating the two rhomboid muscles (major and minor) and become superficial over the medial margin of the scapula. They form the dorsal scapular artery. This superficial branch penetrates the deep side of the trapezius and then gives off several cutaneous perforators that irrigate the skin covering the caudal portion of the trapezius - here it courses 1 to $2 \mathrm{~cm}$ from the muscle's lateral margin. The average diameter of the dorsal scapular artery is $2.7 \mathrm{~mm}$ proximally and at its origin, and $1.95 \mathrm{~mm}$ over the levator scapulae muscle. ${ }^{9}$ A deep branch of the dorsal scapular artery continues its descending trajectory under the rhomboid major muscle.

\section{Materials and methods}

Our work exploited several databases (Medline, Pubmed and Hinari) in order to highlight the most frequently observed anatomical variations on the trapezius muscle. We exploited articles from 1942 to the present day, and selected the most relevant ones. Our work shows that anatomical variations of trapezius muscle, though rare, are widely documented in the literature.

Our methodology consisted of retrospectively listing articles reporting abnormalities of the trapezius muscle. The inclusion criterias were represented by any publication reported by the literature, and describing a unilateral or bilateral congenital anomaly of the trapezius muscle, discovered during clinical examination, surgery, radiological procedures or during cadaveric dissection. As exclusion criterias, we did not take into account publications reporting trapezius muscle abnormalities of accidental or iatrogenic origin. We therefore cited and discussed data from the 15 most relevant publications reporting congenital anomalies of the trapezius muscle.

The main anatomical variations reported in the literature are: partial agenesis of the trapezius, total aplasia or hypoplasia of the trapezius, the presence of accessory muscular portions and anatomical variations on the innervation of the muscle.

\section{Anatomical variations of the trapezius muscle reported by the literature}

Trapezius muscle can have a large morphological diversity. These variations include an abnormal origin or the partial or complete absence of the muscle. Mehra et al. $^{10}$ reported a rare case of a dorsoscapular triangle, due to the bilateral and complete absence of the cervical part of the trapezius muscle. In this case, each side of the muscle was presented as a right angle triangle extending from the $5^{\text {th }}$ cervical vertebrae to the $10^{\text {th }}$ thoracic vertebrae. Both sides of the muscle formed an isosceles triangle instead of a trapezius, hence the name dorsoscapular triangle.

The congenital absence of skeletal muscles is rare, ranging from 0.3 to $1.9 \% .^{10}$ In the case of the trapezius muscle, causes may be inflammatory, vascular, neuropathic and myopathic. The congenital absence of the trapezius muscle maybe part of a Poland syndrome or Klippel-Feil syndrome. ${ }^{11}$

Emsley et al. ${ }^{12}$ have reported a morphological anomaly found during a cadaverous dissection on a 89year-old subject. The left trapezius was significantly smaller than the right trapezius, especially in its caudal part. They also noted that the muscle fibers of the left trapezius were much lighter than on the right. Moreover, the existing fibers of the left trapezius inferior to the scapula were only one-third to two-thirds as thick as those on the right. The vertebral attachment of the inferior fibers of the left trapezius was also notably higher than that on the right. Morphometric analysis indicated that the surface area of the left trapezius was approximately $50 \%$ that of the right trapezius. In addition, the orientation of the fibers was different (Figure 2).

Allouch et al. ${ }^{13}$ reported a total absence of the left trapezius in a 87year-old caucasian. This unilateral agenesis of the trapezius muscle was accompanied by an absence of the left accessory nerve and of the vascular pedicle of the left trapezius.

Hug et al. ${ }^{14}$ also reported a rare anatomical presentation. In a 48-year-old patient with chronic pain and deformities of the right upper limb, they found an accessory portion of the trapezius muscle. This accessory portion crossed the upper part of the brachial plexus, explaining the symptoms on the upper limb. Excision of the anomalous muscle and release of the clavicular part of the sternocleidomastoid muscle abolished the complaints (Figure 3).

Kwak et al. ${ }^{15}$ reported an anatomical variation of the trapezius muscle during the cadaveric dissection of a female Korean subject. They found that the lateral $3 / 4$ of the descending portion of the trapezius were separated from the rest of the muscle. This isolated part of the trapezius was attached to the clavicle by a tendon.

Newman et al. ${ }^{16}$ studied anatomical variations of trapezius in a 5year-old child and his father. Radiographic examination of MRI showed bilateral aplasia of trapezius muscle in children, with clinically bilateral myopathy and camptodactyly. In the father of the 
child, partial and unilateral hypoplasia of the trapezius muscle was found, but without functional repercussions. This hypoplasia was accompanied by a lateral displacement of the right scapula, and a slight bilateral camptodactyly.
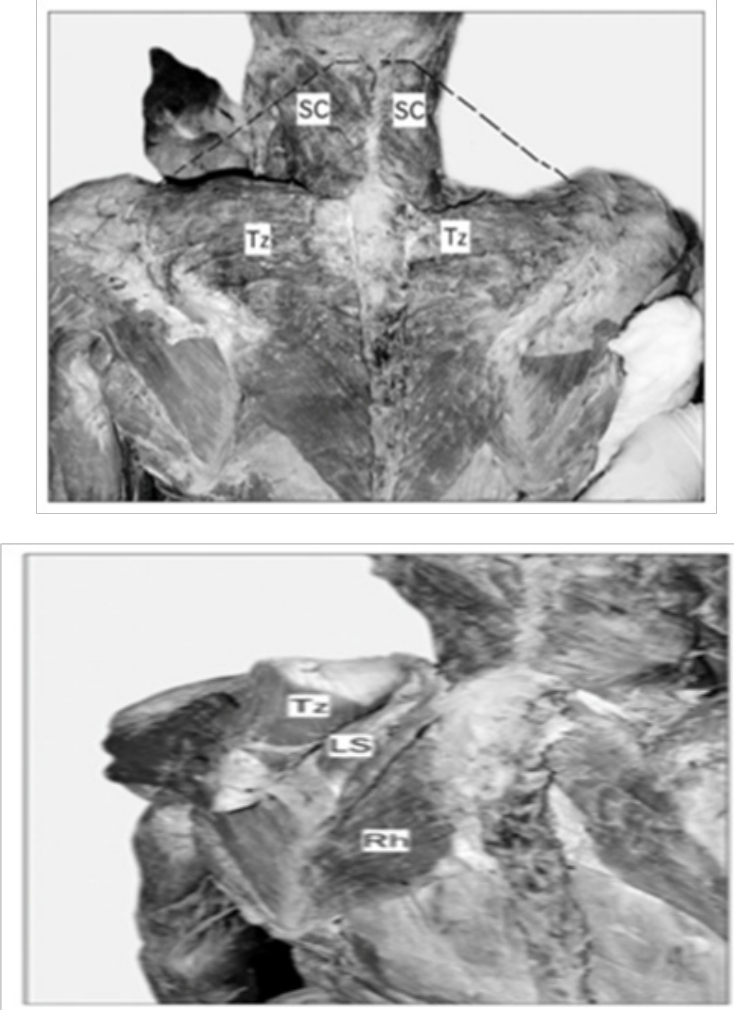

\section{Figure 2}

A) Dissection of the back and neck showing absence of cervical part of trapezius. Dotted black line depicting the normal extent of the cervical part of trapezius. SC, spleniuscapitis; Tz, trapezius.

B) muscles undercover of trapezius seen after reflecting it laterally. LS, levatorscapulae; $\mathrm{Rh}$, rhomboides; $\mathrm{Tz}$, trapezius. ${ }^{10}$

Garbelotti et al ${ }^{17}$ have reported the case of a partial and unilateral absence of the trapezius muscle discovered during dissection of an adult black male cadaver. The detailed examination showed that the ascending part of the trapezius and the descending fibers of the middle part were replaced by a broad $2 \mathrm{~mm}$ thick fascia. Histological analysis showed the presence of a few muscle fibres in the aponeurosis.

From a functional view point, it was possible that this individual would have had difficulty in moving his shoulder, especially during flexion, abduction and circumduction of the arm Figure 4.

Nooij et al. ${ }^{18}$ reported agenesis of the trapezius muscle. During their dissection, the authors noticed that the descending and transverse parts of the trapezius were totally absent. Only a few localized fibers remained on the ascending part of the muscle.

Gross-Kieselstein et al. ${ }^{19}$ also reported a study in which 2 parents had a complete absence of trapezius muscle. The first case is that of a 17year-old teenager presenting with a complete lack of muscular structure on the trapezius in the back region, and on the area of the pectoralis major in front (Figure 5). The second case concerns the older brother of the first case. Aged of 28, he had an absence of trapezius muscles and pectoralis major only on the right side.

Stacey et al. $^{20}$ have reported a case of anatomical variation concerning the accessory nerve. In this case the trapezius muscle was directly innervated by the cervical plexus and received no branch of the accessory nerve. Their work supports the hypothesis that complete paralysis of the trapezius muscle does not occur systematically when the accessory nerve is sacrificed during radical neck dissection.

Beaton et al. ${ }^{21}$ found numerous variations in relation to the original insertions of the trapezius muscle. These variations relate to trapezius insertion anomalies on the vertebrae or the nuchal ligament, left and right trapezius symmetry anomalies, and abnormalities in muscle size.
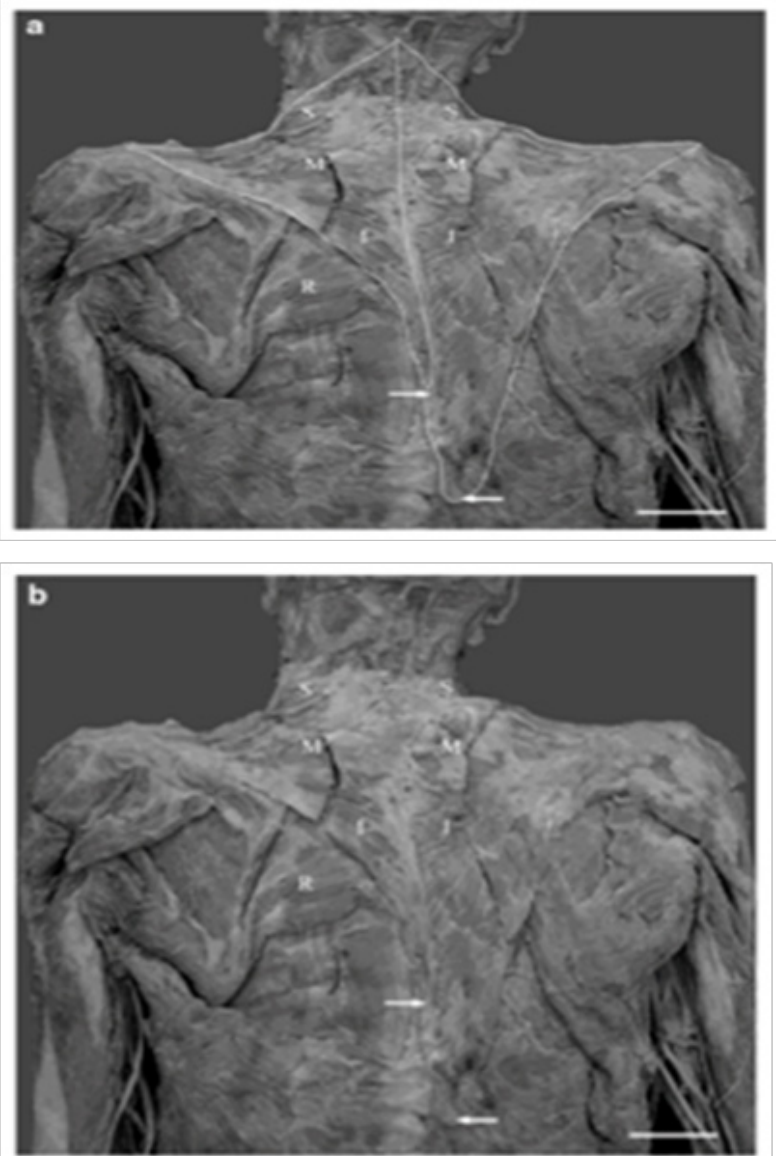

Figure 3

A) Digitized image of a unilaterally hypoplastic trapezius muscle on the left side.

B) Arrows indicate the approximate inferior attachment points of the left and right trapezius muscles. Note the easily visible rhomboideus major muscle $(R)$ on the leftside. Superior (S), middle (M), and inferior (I) segments of trapezius. Scale bar $5 \mathrm{~cm} .^{12}$ 

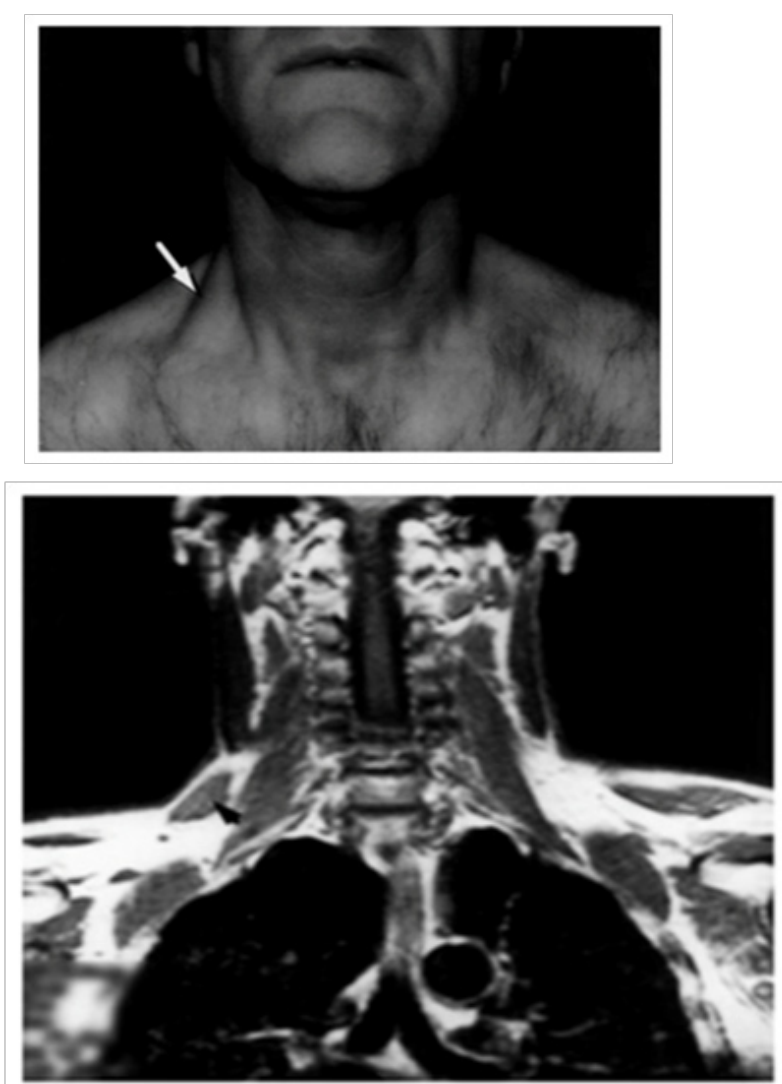

Figure 4

A) Swelling (arrow) in the right posterior triangle of the neck. Frontal magnetic resonance section showing the accessory part of the right trapezius muscle (arrowhead). ${ }^{14}$

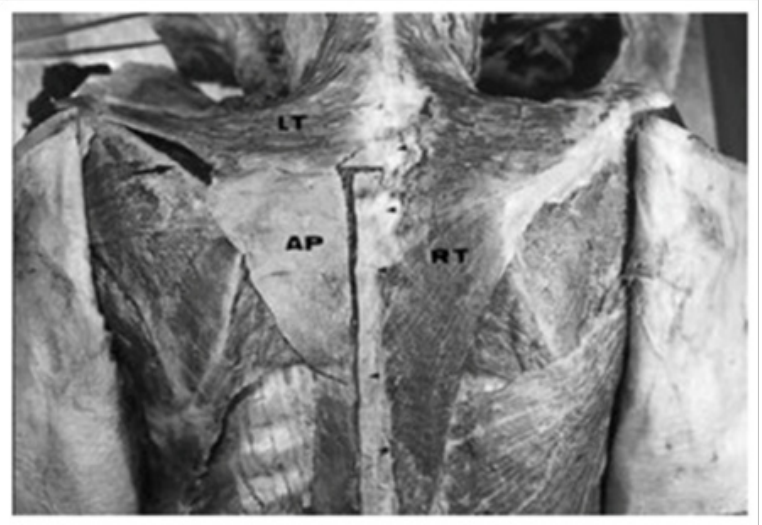

Figure 5 Dorsal view showing the normal right trapezius (RT), the descending fibres and part of the transverse fibres of the left trapezius (LT), and an aponeurosis replacing the ascending fibres of the left trapezius (AP). The aponeurosisattachs to C7 and TI-T6 (short arrows), rhomboid major (RM) and spine of the scapula (long arrow). ${ }^{17}$

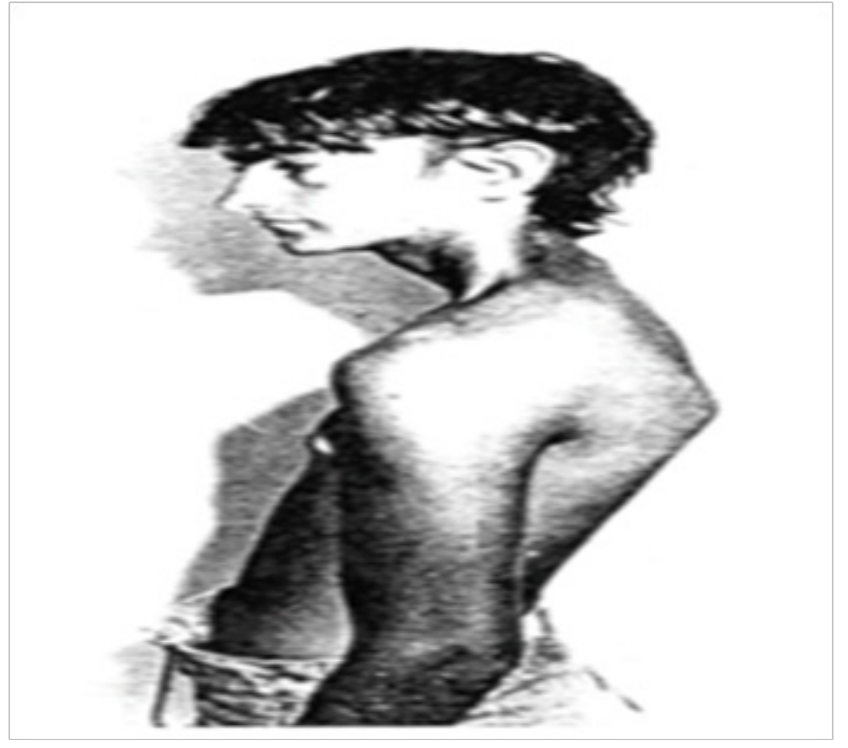

Figure 6 Familial absence of the trapezius muscle with associated shoulder girdle abnormalities. ${ }^{19}$

\section{Discussion}

Apart from the pectoralis major muscle, the trapezius muscle is the one with the most frequent abnormalities in its morphology according to Bing. ${ }^{22}$ However, this assertion has been very little documented in the literature. Trapezius muscle belongs to the muscles of the trunk, it is located in the cranial region of the back. Rarely the trapezius muscle may have abnormalities. These abnormalities can be of clinical discovery, intraoperative, or during cadaveric dissection. These anatomical variations are well documented in the literature. They can have important functional consequences. In addition, the practitioner must take this into account before harvesting any trapezius muscular flap.

The first explanation for these variations is genetic, as reported by Gross ${ }^{19} \&$ Adam..$^{23}$ The second explanation involves the embryological factor. ${ }^{1,24}$ This may explain why it is quite common that patients with anatomical variations of the trapezius muscle also have other malformations (Table 1).

The trapezius muscle develops from the gill musculature that initially appears in the $7 \mathrm{~mm}$ embryo, ventrally to the 2 caudal occipital myotomes and 2 anterior cervical myotomes. This element will then separate in the $9 \mathrm{~mm}$ embryo into a sternocleidomastoid ventral portion and a trapezial dorsal portion. In the $11 \mathrm{~mm}$ embryo the trapezial portion will gradually extend to the $6^{\text {th }}$ pair of cervical nerves. At this stage the muscle is not yet attached to the skeleton of the shoulder, and it is separated from a more dorsal myotomous mass by the deep cervical fascia. In the $16 \mathrm{~mm}$ embryo the muscle will contract its attachments with the spine of the scapula and the clavicle. Cranially it extends to the nuchal ligament, however it does not yet join the occipital cartilage. It is only in the $20 \mathrm{~mm}$ embryo that the muscle will acquire its definitive configuration. ${ }^{25}$ 
Table I Summary table of trapezius muscle abnormalities

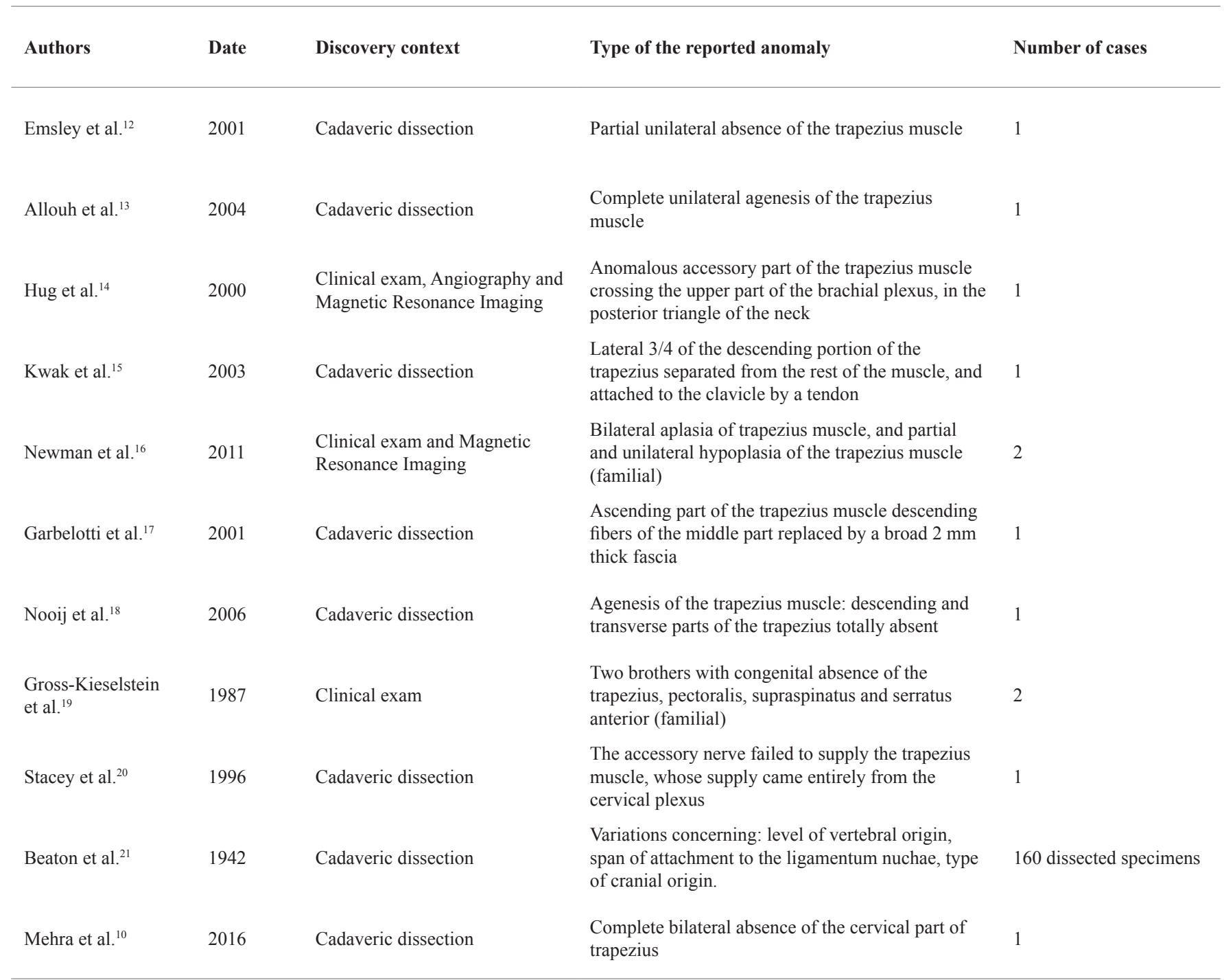

\section{Conclusion}

The anatomical variations of the trapezius muscle are rare in the literature. They are most often fortuitous discovery, and of genetic or embryological origin. Despite their scarcity they can be very varied and have important functional consequences. Even in the absence of clinical consequences, anatomical variations of the trapezius muscle must be taken into account when planning any surgery of this muscular region.

\section{Acknowledgements}

None.

\section{Conflict of interest}

The authors declare that they have no conflicts of interest regarding this study.

\section{References}

1. Leon X, Maranillo E, Quer M, et al. Case report: cleidocervical or levator claviculae muscle. A new embryological explanation as to its origin. $J$ Anat. 1995;187(2):503-504.

2. Noussios G. The variational anatomy of the trapezius muscle: A review of the literature. The Internet Journal of Human Anatomy. 2014:3(1)

3. Badura M, Grzonkowska M, Baumgart M, et al. Quantitative anatomy of the trapezius muscle in the human fetus. Adv Clin Exp Med. 2016;25(4):605-609.

4. Johnson G, Bogduk N, Nowitzke A, et al. Anatomy and actions of the trapezius muscle. Clin Biomech (Bristol, Avon). 1994;9(1):44-50.

5. Mathes SJ, Nahai F. Classification of the vascular anatomy of muscles: experimental and clinical correlation. Plast Reconstr Surg. $1981 ; 67(2): 177-187$.

6. Haas F, Weiglein A, Schwarzl F, et al. The lower trapezius 
musculocutaneous flap from pedicled to free flap: anatomical basis and clinical applications based on the dorsal scapular artery. Plast Reconstr Surg. 2004;113(6):1580-1590.

7. Salmon M, Grégoire R. Artères de la peau. Masson, Paris, France; 1936. p. 123-131.

8. Salmon M, Dor J. Les artères des muscles des membreset du tronc. Masson, Paris, France; 1933. 238p.

9. Sadigh PL, Chang LR, Hsieh CH, eta 1. The trapezius perforator flap: An underused but versatile option in the reconstruction of local and distant soft-tissue defects. Plast Reconstr Surg. 2014;134(3):449e-456e.

10. Mehra L, Tuli A, Raheja S. Dorsoscapularis triangularis: embryological and phylogenetic characterization of a rare variation of trapezius. Anat Cell Biol. 2016;49(3):213-216.

11. Miyamoto RT, Yune HY, Rosevear WH. Klippel-Feil syndrome and associated ear deformities. Am J Otol. 1983;5(2):113-119.

12. Emsley JG, Davis MD. Partial unilateral absence of the trapezius muscle in a human cadaver. Clin Anat. 2001;14(5):383-386.

13. Mohammed Z Allouh, Adel Mohamed, Azedean Mhanni. Complete unilateral absence of trapezius muscle. McGill J Med. 2004;8:31-33.

14. Hug U, Burg D, Meyer VE. Cervical outlet syndrome due to an accessory part of the trapezius muscle in the posterior triangle of the neck. $J$ Hand Surg Br. 2000;25(3):311-313.

15. Kwak HH, Kim HJ, Youn KH, et al. An anatomic variation of the trapezius muscle in a Korean: the cleido-occipitalis cervicalis. Yonsei Med J. 2003;44(6):1098-1100.
16. Newman CJ, Jacquemont S, Theumann N, et al. Familial aplasia of the trapezius muscle: clinical and MRI findings. Acta Paediatri. 2011;100(3):464-466.

17. Garbelotti Júnior SA, Rodrigues CF, Sgrott EA, et al. Unilateral absence of the thoracic part of the trapezius muscle. Surg Radiol Anat. 2001;23(2):131-133.

18. Nooij LS, Oostra RJ. Trapezius aplasia: indications for a dual developmental origin of the trapezius muscle. Clin Anat. 2006;19(6):547-549.

19. Gross-Kieselstein E, Shalev RS. Familial absence of the trapezius muscle with associated shoulder girdle abnormalities. Clin Genet. 1987;32(3):145-147.

20. Stacey RJ, O'Leary ST, Hamlyn PJ. An anomaly in the nerve supply of the trapezius muscle. Clin Anat. 1996;9(6):414-416.

21. Lindsay E Beaton, Barry $\mathrm{J}$ Anson. Variations in the origin of the M. trapezius. The Anatomical Record. 1942;83(1):41-46.

22. Bing R. Ueber angeborene Muskeldefecte. Virchow's Arch fpath Anat 1902;170(2):175-228.

23. Adams SB Jr, Flynn JM, Hosalkar HS, et al. Torticollis in an infant caused by hereditary muscle aplasia. Am J Orthop (Belle Mead NJ). 2003;32(11):556-558

24. Debeer P, Brys P, De Smet L, et al. Unilateral absence of the trapezius and pectoralis major muscle: a variant of Poland syndrome. Genet Couns. 2002;13(4):449-453

25. Keibel F, Mall FP. Manual of human embryology. Philadelphia: JB Lippincott Company; 1910. 